\title{
Review
}

\section{Salmonella-based Anticancer Vaccines and their Efficacy}

\author{
Samad Farashi-Bonab, PhD $^{1 *}$; Nemat Khansari, PhD $^{2}$
}

'Department of Immunology, School of Medicine, Tehran University of Medical Sciences, Tehran, Iran

${ }^{2}$ American Medical Diagnostic Laboratory, Garden Grove, CA 92843, USA

\section{"Corresponding author}

Samad Farashi-Bonab, PhD

PostDoc, Department of Immunology, School of Medicine, Tehran University of Medical Sciences, Tehran, Iran; Tel. +982 I64432465; Fax: +982 I664 I9536;

E-mail: Farashibs@yahoo.com

\section{Article Information}

Received: December 21 st 2018 ; Revised: March 19th 2019 ; Accepted: March 15 ${ }^{\text {th }}$ 2019; Published: April $3^{\text {rd }}$, 2019

\section{Cite this article}

Farashi-Bonab S, Khansari N. Salmonella-based anticancer vaccines and their efficacy. Vaccin Res Open J. 20I9; 4(I): 5-II. doi: I0.I7I40/VROJ-4-III

\section{| ABSTRACT |}

Surgery, chemotherapy, and radiotherapy are successfully used to treat patients with tumors or cancers. However, the innovation of more potent therapeutic modalities is essential for the efficient treatment of patients with advanced cancers. More than two centuries ago, bacteria have been observed to have beneficial effects in some cancer patients. Virulence factors of some bacteria and their infectious behavior in the body suggest their effectiveness in tumor suppression. At present, bacillus calmette-guérin (BCG), a live attenuated strain of Mycobacterium bovis, is currently used to treat bladder cancer. Some other bacteria have also been found to have antitumor activities. Anaerobic bacteria can colonize solid tumors and exert an intrinsic antitumor effect. Salmonella is the most studied bacterium in the field of bacterial anticancer therapy in preclinical studies. In this article, we discuss progress in the development of bacterial anticancer vaccines, especially Salmonella-based vaccines, their antitumor efficacy, and mechanisms involved in vaccine-mediated cancer cell death.
\end{abstract}

\section{Keywords}

Cancer; Vaccine; Bacteria; Salmonella; Apoptosis; Immune responses; Cancer therapy efficacy.

\section{Abbreviations}

MHC: Major Histocompatibility Complex; TGF- $\beta$ : Transforming Growth Factor-Beta; VEGF: Vascular Endothelial Growth Factor; IL: Interleukin; PD: Programmed Cell Death; APCs: Antigen Presenting Cells; PD-L1: PD-Ligand 1; TLRs: Toll-Like Receptors; BCG: Bacillus Calmette-Guérin; CFU: Colony-Forming Units; TNF- $\alpha$ : Tumor Necrosis Factor-Alpha; TRAIL: Tumor Necrosis Factor-Related Apoptosis-Inducing Ligand; PSA: Prostate-Specific Antigen; CTLA-4: Cytotoxic T Lymphocyte Associated Antigen-4, IFN- $\gamma$ : Interferon-Gamma; Th1: T Helper Type 1; Omp A: Outer Membrane Protein A.

\section{INTRODUCTION |}

$\mathrm{H}$ undreds of years ago, variolation has been used to protect humans against smallpox. At the end of the $18^{\text {th }}$ century, the cowpox virus was introduced by Edward Jenner as a safer alternative to the variola virus, even though the virus was not identified at that time. During the $19^{\text {th }}$ century after the discovery of bacteria as causative agents of infectious diseases, attenuated or killed microorganisms or a component of the whole microorganism were widely used to induce immunity against infectious diseases. Use of these vaccines led to a dramatic decrease in death induced by viral and bacterial agents. In recent decades, researchers attempted to develop therapeutic vaccines against cancer with the aim of re- cruiting lymphocytes and other immune cells to destroy cancerous cells. These vaccines were used as a monotherapy or in combination with conventional therapeutic modalities such as surgery, chemotherapy, and radiotherapy to prevent metastases and recurrence of cancer. ${ }^{1}$

T-cells are an important part of protective adaptive immune responses. Immunogenic cancer cell growth can result in the generation of antitumor immune responses, especially T-cell responses. ${ }^{2}$ Intravenous infusion of tumor-sensitized T-cells from immune donors has resulted in regression of large established tumors in T-cell-deficient recipients., ${ }^{3,4}$ High number of intraepithelial $\mathrm{CD}^{+}$tumor-infiltrating T-cells was associated with the 
absence of lymph node metastases in patients with human papillomavirus-positive cervical cancer. ${ }^{5}$ High $\mathrm{CD}^{+} / \mathrm{CD}^{+}{ }^{+}$-cell ratio of tumor-infiltrating lymphocytes was associated with better clinical outcome in colorectal cancer patients. ${ }^{6}$ In contrast, it has been shown that T-cell-mediated suppression of antitumor immunity occurs in progressive growth of an immunogenic tumor. ${ }^{3} \mathrm{In}-$ deed, immunogenic tumor cells can induce $\mathrm{CD}^{+}$T-cell responses, however, tumors can avoid immune cell-mediated destruction by induction of T-cell tolerance as well. ${ }^{7,8}$

Induction of T-cell responses is the major aim of vaccines. Vaccines can produce T-cell responses to a large number of antigens. It is preferable to include more than one antigen in vaccines to decrease the likelihood of immune escape. But, immunological tolerance to cancer cell antigens is a usual phenomenon. Furthermore, tumor cell growth and proliferation can lead to the establishment of tumor tissue with an immunosuppressive tumor microenvironment. ${ }^{9}$ Therefore, breaking immunological tolerance and immunosuppression is necessary for therapeutic cancer vaccines. Tumors employ numerous mechanisms limiting natural or vaccine-induced antitumor immune responses. Some of these mechanisms include down-regulation of major histocompatibility complex (MHC) class I molecules, lack of expression of costimulatory molecules CD80 and CD86, expression of Fas ligand, and secretion of immunosuppressive cytokines such as transforming growth factor beta (TGF- $\beta$ ), vascular endothelial growth factor (VEGF), and Interleukin 10 (IL-10). ${ }^{10,11}$ Tumor infiltrating dysfunctional $\mathrm{CD}^{+}{ }^{+}$T-cells expressing the immune checkpoint molecule programmed cell death 1 (PD-1) have been detected in various types of cancer, ${ }^{12-14}$ which was associated with poor clinical outcome for patients. ${ }^{12}$ Tumor cells and antigen-presenting cells (APCs) expressing PD-L1 can impair proliferation and effector function of PD- $1^{+}$T-cells in the tumor microenvironment. ${ }^{15}$ In clinical trials, blocking PD-1/PD-L1 interactions with anti-PD-1 antibodies led to improved antitumor immunity. ${ }^{16}$ In addition, increased frequency of immunosuppressive cells such as regulatory T-cells has been reported in the peripheral blood and tumor microenvironment of cancer patients. ${ }^{17}$ Depleting these cells or blocking their immunosuppression functions by monoclonal antibodies has resulted in improved antitumor immunity in some types of cancer. ${ }^{18}$ However, more effective therapeutic approaches should be developed to produce potent antitumor immune responses in patients with advanced cancer.

APCs have a crucial role in antitumor immunity as they trigger adaptive immune responses by processing and presenting antigens for recognition by T-cells. ${ }^{19}$ Bacterial products can strongly activate APCs through pattern recognition receptors such as toll-like receptors (TLRs). Some bacterial products have antitumor properties by apoptosis induction in cancer cells. For instance, the bacterial protein azurin, a cupredoxin type of electron transfer and purified redox protein from Pseudomonas aeruginosa, selectively induced apoptosis in human breast cancer cells ${ }^{20}$ and oral squamous carcinoma cells. ${ }^{21}$ This bacterial protein effectively enters human cancer cells, but not normal cells, ${ }^{22}$ and it has been shown to induce apoptosis through stabilization of the tumor suppressor protein p53. ${ }^{23}$ In some studies, bacterial products have been used as an ad- juvant for anticancer vaccines. ${ }^{24}$ In addition, live bacteria expressing tumor antigen have been used for tumor antigen delivery in vivo. ${ }^{25,26}$ These therapeutic vaccination approaches did not lead to the eradication of established experimental tumors or human tumors. Nonetheless, some live bacteria have been successfully used for cancer treatment. ${ }^{27}$

\section{APPLICATION OF BACTERIA IN ANTICANCER THERAPY -}

More than two centuries ago, cancer remission was reported in cancer patients after recovering from bacterial infections. In the late $19^{\text {th }}$ century, William Coley used live and heat-killed bacteria such as Streptococcus pyogenes and Serratia marcescens to treat cancer patients. The Coley's heat killed bacteria, known as Coley's toxin, has been used for sarcoma patients until 1963. In the mid-1980s, Bacillus Calmette-Guérin (BCG), a live attenuated strain of $M y$ cobacterium bovis, was used to treat superficial bladder cancer. ${ }^{27} \mathrm{At}$ present, BCG is a common treatment for bladder cancer and it is the only bacterial agent approved by the US Food and Drug Administration (FDA) for primary therapy of carcinoma in situ of the bladder. BCG is successfully used to treat some non-invasive bladder cancers two weeks after surgery. ${ }^{28}$ Some other bacteria have also been found to have antitumor activities.

Almost 70-years ago, it has been shown that anaerobic bacteria can selectively grow in tumors. ${ }^{29,30}$ Several species of anaerobic bacteria have the ability to colonize solid tumors and induce tumor shrinkage. Colonization of anaerobic bacteria in solid tumors is largely due to impaired blood flow and extensive necrosis in the central part of tumor tissue. Selective localization of bacteria in chemically-induced rat mammary tumors was observed after systemic injection of Bifidobacterium longum, a nonpathogenic, anaerobic bacterium. ${ }^{31}$ It has also been shown that obligate anaerobic bacteria Clostridium novyi and Clostridium sordelli localize solid tumors, especially in the hypoxic parts of tumor tissues. ${ }^{32}$ However, small tumors, as well as metastatic tumors, may not be targeted by obligate anaerobic bacteria as these regions have sufficient blood circulation and are not hypoxic. In experimental syngeneic and allogeneic murine tumor models using light-emitting bacteria, several facultative anaerobic bacterial species such as Escherichia coli, Salmonella enterica serovar Typhimurium (S. typhimurium), Vibrio cholerae, and Listeria monocytogenesis were able to selectively enter solid tumors and metastases and replicate within tumors. ${ }^{33,34}$ Intravenously injected facultative anaerobic bacteria can enter and replicate within tumors with different efficacy. It has been shown that Escherichia coli robustly replicates in tumor while Streptococcus pyogenes shows a much lower level of replication in the tumor. Marine bacteria Photobacterium phosphoreum and Vibrio fischeri did not show tumor-specific colonization after intravenous injection. The colonization process was independent of the tumor type but largely dependent on the stage of tumor development. ${ }^{35}$ The majority of injected bacteria have been found in the central part of the tumors. This part of large tumors is usually necrotic. Moreover, the bacterial dose can affect the colonization of tumor, ${ }^{35}$ as a small number of bacteria may be cleared by the body's immune system. Thus, an appropriate number of bacteria with antitumor properties are required to overcome the immune clearance mechanisms in order 
to survive during tumor entry.

\section{SALMONELLA-BASED ANTICANCER VACCINES, THEIR THERAPEUTIC EFFICACY,AND MECHANISMS INVOLVED IN CANCER CELL DEATH -}

Salmonella is a gram-negative, facultative anaerobe, and a pathogen to human and animals. This bacterium is extensively studied in the field of bacterial therapy of cancer. Some Salmonella strains preferentially colonize solid tumors and show an intrinsic antitumor effect. S. typhimurium strains exhibited high tumor colonization following systemic administration into tumor-bearing mice, resulting in more than $1 \times 108 \mathrm{CFU}$ per gram tumor tissue. ${ }^{36}$ After intravenous administration of $S$. typhimurium, bacteria were found in blood, spleen, and liver. Low numbers of bacteria were detected in tumors associated with blood vessels. A rapid increase of the proinflammatory cytokine TNF- $\alpha$ was detected in the blood which was linked to a tremendous influx of blood into tumors by vascular disruption, resulting in bacteria flushing into the tumor. Blood influx was followed by necrosis formation, bacterial growth and infiltration of neutrophils. ${ }^{37}$ Bacterial motility or chemotactic responsiveness have not been required for tumor invasion and colonization of Salmonella. ${ }^{38}$ Both intravenous and intraperitoneal injection of S. typhimurium led to complete tumor clearance. In contrast, after oral administration, tumor colonization was transient, inefficient, and delayed with no therapeutic effect observed. ${ }^{38}$ Furthermore, oral infection with Salmonella may increase the risk of gall-bladder cancer $^{39}$ and colon cancer. ${ }^{40}$ This pathogen also has the potential for causing sepsis. However, tumor-colonizing Salmonella can be readily controlled by systemic administration of antibiotics. ${ }^{41}$

Attenuation of Salmonella strains has been performed by genetic alterations to prevent bacteria-induced septic shock, especially by lipopolysaccharide deletion. ${ }^{42,43}$ Several genetically modified Salmonella strains have been developed such as VNP20009, LT2, CRC1674, A1-R, and CRC2631. ${ }^{43-46}$ Intravenous administration of attenuated S. typhimurium, VNP20009, has been evaluated in phase I clinical trial for the treatment of 24-patients with nonresponsive metastatic melanoma and one patient with renal cell carcinoma. Dose-limiting toxicity, including thrombocytopenia, anemia, persistent bacteremia, hyperbilirubinemia, diarrhea, vomiting, nausea, elevated alkaline phosphatase, and hypophosphatemia, was observed in patients receiving $1 \times 109 \mathrm{CFU} / \mathrm{m}^{2}$. VPN2009 induced a dose-related increase in the circulation of proinflammatory cytokines IL-1 beta, TNF- $\alpha$, IL-6, and IL-12. Focal tumor colonization was observed in two patients receiving $1 \times 109 \mathrm{CFU} / \mathrm{m}^{2}$ and in one patient receiving $3 \times 108 \mathrm{CFU} / \mathrm{m}^{2}$. Objective tumor regression was not observed in the patients, including those patients with colonized tumors. ${ }^{47}$ The genetically modified, amino acid auxotrophs, S. typhimurium A1 strain grew throughout the tumor, including viable malignant tissue. The bacteria invaded and replicated intracellularly in PC-3 prostate cancer cells grafted into nude mice and caused tumor inhibition and regression. Normal tissue was cleared of these bacteria even in immunodeficient athymic mice. ${ }^{45} \mathrm{~S}$. typhimurium A1 was also able to inhibit PC-3 human prostate cancer experimental bone metastasis and significantly improved the overall survival of mice with multiple bone metastases. ${ }^{48}$
In a mouse infection model, $S$. typhimurium mutants from which Braun lipoprotein genes (lppA and B) and the multicopy repressor of high-temperature requirement (msbB) gene were deleted a minimally induced proinflammatory cytokine production was observed. Immunization with these mutants followed by challenge with the wild-type $S$. typhimurium significantly inhibited tumor growth, as $88 \%$ regression in tumor size was observed in ippB $/ \mathrm{msbB}$ mutant immunized mice. The tumor size regression was correlated to downregulation of CD44 high and CD $4^{+} \mathrm{CD} 25^{\mathrm{s}}$ regulatory T-cells. ${ }^{49}$ Tumors are a rich source of purines with adenosine triphosphate concentrations. A Salmonella strain deficient in synthesizing purines by a mutation in the purl gene which encodes for purine biosynthetic enzyme has been used with tumor-specific localization. ${ }^{50}$ Live attenuated $S$. typhimurium genetically modified at purl and msbB genes (VNP20009 strain) when administrated intravenously to tumor-bearing mice inhibited the growth of tumors, which did not depend on the presence of $\mathrm{T}$ and B-cells. ${ }^{51}$ Increased number of bacteria was detected in the tumor. The bacterial number reached levels 10,000 times higher than in the normal liver reservoir. Salmonella growth was observed in areas of the tumor which partially inhibit tumor growth. However, a rim of tumor survived and resulted in progressive tumor growth. ${ }^{52}$

Orally administration of attenuated $S$. typhimurium carrying a eukaryotic expression vector that contains the second-derived activator of caspases (Smac) and TRAIL genes under the control of the human telomerase reverse transcriptase promoter inhibited tumor growth by $70-90 \%$ and prolonged the survival of mice. ${ }^{53}$ Immunization of mice with recombinant $S$. typhimurium expressing C-Raf significantly reduced tumor growth in transgenic mouse models of Rafoncogen-induced lung adenomas. ${ }^{54}$ Vaccination with recombinant attenuated $S$. typhimurium aroA strain secreting prostate-specific antigen (PSA) and cholera toxin subunit B induced cytotoxic $\mathrm{CD} 8^{+} \mathrm{T}$-cell responses and efficient prevention of tumor growth in mice. ${ }^{55}$ Intravenous injection of attenuated $S$. typhimurium strains reduced immunosuppression in the tumor and tumor-draining lymph node. ${ }^{56,57}$ However, injection of unmodified $S$. typhimurium or genetically-modified $S$. typhimurium expressing recombinant tumor proteins did not result in eradication of long-established tumors in mice.

Immunogenic melanoma tumors can grow progressively even when the tumor is infiltrated by $\mathrm{CD}^{+} \mathrm{T}$-cells. Long-established immunogenic tumors have been shown to contain a high percentage of PD- $1^{+}$tumor-specific $\mathrm{CD} 8^{+} \mathrm{T}$-cells. Treatment with PD-L1 and CTLA-4 blocking antibodies was ineffective in preventing the growth of progressive tumors. Exogenous tumor-specific antigen delivery into tumors using $S$. typhimurium expressing a recombinant antigen resulted in induction of proliferation of tumor-specific $\mathrm{CD} 8^{+} \mathrm{T}$-cells in the lymphoid organs and recovered effector function of tumor-specific $\mathrm{CD}^{+} \mathrm{T}$-cells in the tumor. Immunization with this vaccine led to improved mice survival and rejection of $32 \%$ of long-established immunogenic melanoma tumors. However, following the treatment, the majority of tumor-specific $\mathrm{CD}^{+}{ }^{+}$T-cells expressed a high-level of PD-1 in the tumor. Combination of injection of $S$. typhimurium expressing the recombinant antigen with programmed cell death-ligand 1 (PD- 
L1) blocking antibody enhanced the expansion of tumor-specific CD $8^{+}$T-cells and resulted in $80 \%$ tumor rejection. ${ }^{58}$ Genetically modified $S$. typhimurium harboring short hairpin RNA against inhibin alpha subunit caused remarkable cytotoxicity in cancer cells compared with unmodified S. typhimurium. This tumor-targeted therapy also significantly inhibited the growth of colon cancers and melanomas and prolonged the survival of mice bearing syngeneic tumors. ${ }^{59}$

Genetically modified $S$. typhimurium A1 has been shown to grow in the cytoplasm of PC-3 human prostate cancer cells and caused nuclear destruction in vitro. The bacteria, introduced intravenously or intratumorally, caused tumor inhibition and regression of xenografts in nude mice. S. typhimurium A1 was undetectable in the liver, lung, spleen, and kidney, but it continued to proliferate in the PC-3 tumor. Intratumoral injection of the bacteria resulted in complete tumor regression by day $20 .{ }^{45}$ Preferential destruction of mitochondria has been observed $8 \mathrm{~h}$ after inoculation of genetically modified Salmonella in PC-3M human prostate cancer cells, but, the nucleus was not apparently affected by Salmonella within 8 h. ${ }^{46}$ Salmonella invasions can induce apoptosis in infected cells as apoptosis has been observed in Salmonella-infected macrophages ${ }^{60,61}$ and intestinal epithelial cells. ${ }^{62}$ Furthermore, apoptosis of Salmonella-infected cancer cells can result in antitumor immune responses through triggering cross-presentation of tumor antigens on $\mathrm{MHC}$ class I molecules of professional antigen presenting cells to cytotoxic T-cells. ${ }^{63}$

Infection with Salmonella results in activation of both innate and adaptive immune responses. This bacterium induces production of numerous cytokines such as IL-1 $\beta$, IL-6, TNF- $\alpha$, IFN- $\gamma$, and IL-12 and recruits and activates APCs such as dendritic cells. Importantly, Salmonella can trigger Th1 polarization which is favorable to antitumor immune responses. S. typhimurium outer membrane protein A (OmpA) induces the maturation of tumor antigen-pulsed dendritic cells resulting in IL-12 production and generation of Th1 immune responses. Bone marrow-derived dendritic cells stimulated with OmpA of $S$. typhimurium generated effective antitumor immunity in a mouse tumor model. ${ }^{64}$ Salmonella-based anticancer vaccines reducing the frequencies or functions of immunosuppressor cells, such as regulatory T-cells and myeloid-derived suppressor cells, may show improved efficacy in cancer patients. Intratumoral injection of attenuated $S$. typhimu rium significantly inhibited Her-2/neu-expressing tumor growth which was associated with increased levels of $\mathrm{TNF}-\alpha$-secreting neutrophils $\left(\mathrm{CD} 11 \mathrm{~b}^{+} \mathrm{Gr} 1^{+}\right.$myeloid cells) and reduced levels of $\mathrm{CD} 4^{+} \mathrm{CD} 25^{+}$Foxp $^{+}$regulatory T-cells in vaccinated mice. ${ }^{65}$

\section{CONCLUSION}

Salmonella is the most studied bacterium for developing a bacterial anticancer therapeutic vaccine. This bacterium is an intracellular microorganism and can induce Th1 immune responses and other antitumor immune cells. Furthermore, Salmonella preferentially colonizes solid tumors and exhibits an intrinsic antitumor effect. Vaccination with $S$. typhimurium strains resulted in therapeutic outcomes in several preclinical studies. However, enhancing their therapeutic efficacy is essential for clinical application in cancer patients. Elucidation of Salmonella-mediated mechanisms in cancer cell destruction can lead to improved bacterial anticancer therapy by utilization of bacterial strains with potent cytotoxic activities against cancer cells. Increasing our understanding of the tumor cell-specific metabolisms may also be beneficial to identify tumor cell metabolic demands and metabolic final products in the tumor microenvironment to find more appropriate bacterial strains with desired metabolic pathways for tumor-selective colonization and destruction in cancer patients.

\section{CONFLICTS OF INTEREST}

The authors declare that they have no conflicts of interest.

\section{REFERENCES}

1. Farashi-Bonab S, Khansari N. Anticancer dendritic cell vaccines producing effective antitumor T-cell responses. Int $J$ V accines Vaccin. 2017; 4(3): 00081.doi: 10.15406/ijvv.2017.04.00081

2. Dye ES. The antimetastatic function of concomitant antitumor immunity. II. Evidence that the generation of $\mathrm{Ly}-1^{+} 2^{+}$effector Tcells temporarily causes the destruction of already disseminated tumor cells. J Immunol. 1986; 136(4): 1510-1515.

3. Berendt MJ, North RJ. T-cell-mediated suppression of anti-tumor immunity. An explanation for progressive growth of an immunogenic tumor. J Exp Med. 1980; 151(1): 69-80.

4. Dye ES, North RJ. Specificity of the T-cells that mediate and suppress adoptive immunotherapy of established tumors. J Lenkoc Biol. 1984; 36(1): 27-37.

5. Piersma SJ, Jordanova ES, van Poelgeest MI, et al. High number of intraepithelial $\mathrm{CD}^{+}$tumor-infiltrating lymphocytes is associated with the absence of lymph node metastases in patients with large early stage cervical cancer. Cancer Res. 2007; 67(1): 354-361. doi: 10.1158/0008-5472.CAN-06-3388

6. Diederichsen AC, Hjelmborg JB, Christensen PB, Zeuthen $\mathrm{J}$, Fenger C. Prognostic value of the $\mathrm{CD}^{+} / \mathrm{CD}^{+}$ratio of tumourinfltrating lymphocytes in colorectal cancer and HLA-DR expression on tumour cells. Cancer Immunol Immunother. 2003; 52(7): 423-428. doi: 10.1007/s00262-003-0388-5

7. Willimsky G, Blankenstein T. Sporadic immunogenic tumours avoid destruction by inducing T-cell tolerance. Nature. 2005; 437(7055): 141-146. doi: 10.1038/nature03954

8. Kline J, Brown IE, Zha YY, et al. Homeostatic proliferation plus regulatory T-cell depletion promotes potent rejection of B16 melanoma. Clin Cancer Res. 2008; 14(10): 3156-3167. doi: 10.1158/10780432.CCR-07-4696

9. Singh S, Ross SR, Acena M, Rowley DA, Schreiber H. Stroma is critical for preventing or permitting immunological destruction of 
antigenic cancer cells. J Exp Med. 1992; 175(1): 139-146.

10. Khong HT, Restifo NP. Natural selection of tumor variants in the generation of "tumor escape" phenotypes. Nat Immunol. 2002; 3(11): 999-1005. doi: 10.1038\%2Fni1102-999

11. Mapara MY, Sykes M. Tolerance and cancer: mechanisms of tumor evasion and strategies for breaking tolerance. J Clin Oncol. 2004; 22(6): 1136-1151. doi: 10.1200/JCO.2004.10.041

12. Thompson RH, Dong H, Lohse CM, et al. PD-1 is expressed by tumor-infiltrating immune cells and is associated with poor outcome for patients with renal cell carcinoma. Clin Cancer Res. 2007; 13(6): 1757-1761. doi: 10.1158/1078-0432.CCR-06-2599

13. Yamamoto R, Nishikori M, Kitawaki T, et al. PD-1-PD-1 ligand interaction contributes to immunosuppressive microenvironment of Hodgkin lymphoma. Blood. 2008; 111(6): 3220-3224. doi: 10.1182/blood-2007-05-085159

14. Ahmadzadeh M, Johnson LA, Heemskerk B, et al. Tumor antigen-specific CD8 T-cells infiltrating the tumor express high levels of PD-1 and are functionally impaired. Blood. 2009; 114(8): $1537-$ 1544. doi: 10.1182/blood-2008-12-195792

15. Taube JM, Anders RA, Young GD, et al. Colocalization of inflammatory response with B7-h1 expression in human melanocytic lesions supports an adaptive resistance mechanism of immune escape. Sci Transl Med. 2012; 4(127): 127ra37. doi: 10.1126/scitranslmed.3003689

16. Topalian SL, Hodi FS, Brahmer JR, et al. Safety, activity, and immune correlates of anti-PD-1 antibody in cancer. N Engl J Med. 2012; 366(26): 2443-2454. doi: 10.1056/NEJMoa1200690

17. Farashi-Bonab S, Khansari N. Regulatory T-cells in cancer patients and their roles in cancer development/progression. MOJ Immunol. 2014; 1(4): 00024. doi: 10.15406/moji.2014.01.00024

18. Farashi-Bonab S, Khansari N. Improving of antitumor immunity and therapeutic efficacy of cancer vaccines and adoptive immunotherapies using monoclonal antibodies. MOJ Immunol. 2015; 2(5): 00062. doi: 10.15406/moji.2015.02.00062

19. Farashi-Bonab S, Khansari N. Dendritic cell maturation is a critical step in dendritic cell vaccine preparation for cancer therapy. Vaccin Res Open J. 2016; 1(1): 25-32.

20. Punj V, Bhattacharyya S, Saint-Dic D, et al. Bacterial cupredoxinazurin as an inducer of apoptosis and regression in human breast cancer. Oncogene. 2004; 23(13): 2367-2378. doi: 10.1038/ sj.onc. 1207376

21. Cho JH, Lee MH, Cho YJ, et al. The bacterial protein azurin enhances sensitivity of oral squamous carcinoma cells to anticancer drugs. Yonsei Med J. 2011; 52(5): 773-778. doi: 10.3349/ ymj.2011.52.5.773
22. Yamada T, Fialho AM, Punj V, et al. Internalization of bacterial redox protein azurin in mammalian cells: Entry domain and specificity. Cell Microbiol. 2005; 7(10): 1418-1431. doi: 10.1111/j.14625822.2005.00567.x

23. Yamada T, Goto M, Punj V, et al. The bacterial redox protein azurin induces apoptosis in J774 macrophages through complex formation and stabilization of the tumor suppressor protein p53. Infect Immun. 2002; 70(12): 7054-7062. doi: 10.1128\%2FIAI.70.12.7054-7062.2002

24. Cluff CW. Monophosphoryl lipid A (MPL) as an adjuvant for anti-cancer vaccines: Clinical results. Adv Exp Med Biol. 2010; 667:111-123. doi: 10.1007/978-1-4419-1603-7_10

25. Nishikawa H, Sato E, Briones G, et al. In vivo antigen delivery by a Salmonella typhimurium type III secretion system for therapeutic cancer vaccines. J Clin Invest. 2006; 116(7): 1946-1954. doi: 10.1172/JCI28045

26. Le DT, Brockstedt DG, Nir-Paz R, et al. A live-attenuated Listeria vaccine (ANZ-100) and a live-attenuated Listeria vaccine expressing mesothelin (CRS-207) for advanced cancers: phase I studies of safety and immune induction. Clin Cancer Res. 2012; 18(3): 858-868. doi: 10.1158/1078-0432.CCR-11-2121

27. Morales A, Eidinger D, Bruce AW. Intracavitary bacillus calmette-guerin in the treatment of superficial bladder tumors. $J$ Urol. 1976; 116(2): 180-183.

28. Rayn KN, Hale GR, Grave GP, Agarwal PK. New therapies in nonmuscle invasive bladder cancer treatment. Indian J Urol. 2018; 34(1): 11-19. doi: 10.4103\%2Fiju.IJU_296_17

29. Parker RC, Plummer HC, Siebenmann CO, Chapman MG. Effect of histolyticus infection and toxin on transplantable mouse tumors. Proc Soc Exp Biol Med. 1947; 66(2): 461-467.

30. Malmgren RA, Flanigan CC. Localization of the vegetative form of clostridium tetani in mouse tumors following intravenous spore administration. Cancer Res.1955; 15(7): 473-478.

31. Yazawa K, Fujimori M, Nakamura T, et al. Bifidobacterium longum as a delivery system for gene therapy of chemically induced rat mammary tumors. Breast Cancer Res Treat. 2001; 66(2): 165-170.

32. Dang LH, Bettegowda C, Huso DL, Kinzler KW, Vogelstein B. Combination bacteriolytic therapy for the treatment of experimental tumors. Proc Natl Acad Sci U S A. 2001; 98(26): 15155-15160. doi: $10.1073 /$ pnas. 251543698

33. Yu YA, Timiryasova T, Zhang Q, Beltz R, Szalay AA. Optical imaging: Bacteria, viruses, and mammalian cells encoding lightemitting proteins reveal the locations of primary tumors and metastases in animals. Anal Bioanal Chem. 2003; 377(6): 964-972. doi: 10.1007/s00216-003-2065-0 
34. Yu YA, Shabahang S, Timiryasova TM, et al. Visualization of tumors and metastases in live animals with bacteria and vaccinia virus encoding light-emitting proteins. Nature Biotech. 2004; 22(3): 313-320. doi: 10.1038/nbt937

35. Yu YA, Zhang Q, Szalay AA. Establishment and characterization of conditions required for tumor colonization by intravenously delivered bacteria. Biotechnol Bioeng. 2008; 100(3): 567-578. doi: 10.1002/bit.21785

36. Stritzker J, Weibel S, Hill PJ, et al. Tumor-specific colonization, tissue distribution, and gene induction by probiotic Escherichia coli Nissle 1917 in live mice. Int J Med Microbiol. 2007; 297(3): 151-162. doi: 10.1016/j.ijmm.2007.01.008

37. Leschner S, Westphal K, Dietrich N, et al. Tumor invasion of Salmonella enteric serovar Typhimurium is accompanied by strong hemorrhage promoted by TNF-alpha. PLoS One. 2009; 4(8): e6692. doi: 10.1371/journal.pone.0006692

38. Crull K, Bumann D, Weiss S. Influence of infection route and virulence factors on colonization of solid tumors by Salmonella enterica serovar Typhimurium. FEMS Immunol Med Microbiol. 2011; 62(1): 75-83. doi: 10.1111/j.1574-695X.2011.00790.x

39. Nagaraja V, Eslick GD. Systematic review with meta-analysis: the relationship between chronic Salmonella typhi carrier status and gall-bladder cancer. Aliment Pharmacol Ther. 2014; 39(8): 745-750. doi: $10.1111 /$ apt.12655

40. Mughini-Gras L, Schaapveld M, Kramers J, et al. Increased colon cancer risk after severe Salmonella infection. PLoS One. 2018; 13(1): e0189721. doi: 10.1371/journal.pone.0189721

41. Crull K, Weiss S. Antibiotic control of tumor-colonizing Salmonella enterica serovar Typhimurium. Exp Biol Med (Maywood). 2011; 236(11): 1282-1290. doi: 10.1258/ebm.2011.011111

42. Low KB, Ittensohn M, Le T, et al. Lipid A mutant Salmonella with suppressed virulence and TNF-alpha induction retain tumor-targeting in vivo. Nat Biotechnol. 1999; 17(1): 37-41. doi: $10.1038 / 5205$

43. Frahm M, Felgner S, Kocijancic D, et al. Efficiency of conditionally attenuated Salmonella enterica serovar typhimurium in bacterium-mediated tumor therapy. MBio. 2015; 6(2). pii:e00254pii:e00315. doi: 10.1128/mBio.00254-15

44. Clairmont C, Lee KC, Pike J, et al. Biodistribution and genetic stability of the novel antitumor agent VNP20009, a genetically modified strain of Salmonella typhimurium. J Infect Dis. 2000; 181(6): 1996-2002.

45. Zhao M, Yang M, Li XM, et al. Tumor-targeting bacterial therapy with amino acid auxotrophs of GFP-expressing Salmonella typhimurium. Proc Natl Acad Sci USA. 2005; 102(3): 755-760. doi: $10.1073 /$ pnas.0408422102
46. Zhong Z, Kazmierczak RA, Dino A, et al. Salmonella-hos'Tcell interactions, changes in hosT-cell architecture, and destruction of prostate tumor cells with genetically altered Salmonella. Microsc Microanal. 2007; 13(5): 372-383. doi: 10.1017/\$1431927607070833

47. Toso JF, Gill VJ, Hwu P, et al. Phase I study of the intravenous administration of attenuated Salmonella typhimurium to patients with metastatic melanoma. J Clin Oncol. 2002; 20(1): 142-152. doi: 10.1200/JCO.2002.20.1.142

48. Toneri M, Miwa S, Zhang Y, et al. Tumor-targeting Salmonella typhimurium A1-R inhibits human prostate cancer experimental bone metastasis in mouse models. Oncotarget. 2015; 6(31): 3133531343. doi: 10.18632/oncotarget.5866

49. Liu T, Chopra AK. An enteric pathogen Salmonella enterica serovar Typhimurium suppresses tumor growth by downregulating CD44high and CD4 $\mathrm{T}$ regulatory (Treg) cell expression in mice: the critical role of lipopolysaccharide and Braun lipoprotein in modulating tumor growth. Cancer Gene Ther. 2010; 17(2): 97-108. doi: $10.1038 /$ cgt. 2009.58

50. Di Virgilio F. Purines, purinergic receptors, and cancer. Cancer Res. 2012; 72(21): 5441-5447. doi: 10.1158/0008-5472.CAN-121600

51. Luo X, Li Z, Lin S, et al. Antitumor effect of VNP20009, an attenuated Salmonella, in murine tumor models. Oncol Res. 2001; 12(11-12): 501-508.

52. Rosenberg SA, Spiess PJ, Kleiner DE. Antitumor effects in mice of the intravenous injection of attenuated Salmonella typhimurium. J Immunother. 2002; 25(3): 218-225. doi: 10.1097\%2F01. CJI.0000014623.45316.93

53. Fu W, Chu L, Han X, Liu X, Ren D. Synergistic antitumoral effects of human telomerase reverse transcriptase-mediated dualapoptosis-related gene vector delivered by orally attenuated Salmonella enterica serovar typhimurium in murine tumor models. J Gene Med. 2008; 10(6): 690-701. doi: 10.1002/jgm.1191

54. Gentschev I, Fensterle J, Schmidt A, et al. Use of a recombinant Salmonella enterica serovar typhimurium strain expressing CRaf for protection against C-Raf induced lung adenoma in mice. BMC Cancer. 2005; 5: 15. doi: 10.1186/1471-2407-5-15

55. Fensterle J, Bergmann B, Yone CL, et al. Cancer immunotherapy based on recombinant Salmonella enterica serovar typhimurium aroA strains secreting prostate-specific antigen and cholera toxin subunit B. Cancer Gene Ther. 2008; 15(2):85-93. doi: 10.1038/ sj.cgt. 7701109

56. Avogadri F, Mittal D, Saccheri F, et al. Intra-tumoral Salmonella typhimurium induces a systemic anti-tumor immune response that is directed by low-dose radiation to treat distal disease. Eur J Immunol. 2008; 38(7): 1937-1947. doi: 10.1002/eji.200738035 
57. Saccheri F, Pozzi C, Avogadri F, et al. Bacteria-induced gap junctions in tumors favor antigen cross-presentation and antitumor immunity. Sci Transl Med. 2010; 2(44): 44ra57. doi: 10.1126/ scitranslmed.3000739

58. Binder DC, Engels B, Arina A, et al. Antigen-specific bacterial vaccine combined with anti-PD-L1 rescues dysfunctional endogenous T-cells to reject long-established cancer. Cancer Immunol Res. 2013; 1(2): 123-133. doi: 10.1158/2326-6066.CIR-13-0058

59. Yoon W, Yoo Y, Chae YS, Kee SH, Kim B. Therapeutic advantage of genetically engineered Salmonella typhimurium carrying short hairpin RNA against inhibin alpha subunit in cancer treatment. Ann Oncol. 2018; 29(9): 2010-2017. doi: 10.1093/annonc/mdy240

60. Monack DM, Raupach B, Hromockyj AE, Falkow S. Salmonella typhimurium invasion induces apoptosis in infected macrophages. Proc Natl Acad Sci U S A. 1996; 93(18): 9833-9838.

61. Hersh D, Monack DM, Smith MR, et al. The Salmonella invasion $\operatorname{SipB}$ induces macrophage apoptosis by binding to caspase
1. Proc Natl Acad Sci USA. 1999; 96(5): 2396-2401.doi: 10.1073/ pnas.96.5.2396

62. Kim JM, Eckmann L, Savidge TC, et al. Apoptosis of human intestinal epithelial cells after bacterial invasion. J Clin Invest. 1998; 102(10): 1815-1823. doi: 10.1172/JCI2466

63. Kepp O, Tesniere A, Schlemmer F, et al. Immunogenic cell death modalities and their impact on cancer treatment. Apoptosis. 2009; 14(4): 364-375. doi: 10.1007/s10495-008-0303-9

64. Jang MJ, Kim JE, Chung YH, et al. Dendritic cells stimulated with outer membrane protein A (OmpA) of Salmonella typhimurium generate effective anti tumor immunity. Vaccine. 2011; 29(13): 2400-2410. doi: 10.1016/j.vaccine.2011.01.036

65. Hong EH, Chang SY, Lee BR, et al. Intratumoral injection of attenuated Salmonella vaccine can induce tumor microenvironmental shift from immune suppressive to immunogenic. Vaccine. 2013; 31(10): 1377-1384. doi: 10.1016/j.vaccine.2013.01.006 